\title{
Examples of discrete groups of hyperbolic motions conservative but not ergodic at infinity
}

\author{
MASAHIKO TANIGUCHI \\ Department of Mathematics, Kyoto University, Japan
}

(Received 24 September 1987 and revised 18 December 1987)

Abstract. For every $n \geq 2$, a discrete subgroup of isometries of the hyperbolic $n$-space, which is conservative but not ergodic on the sphere at infinity, is constructed.

\section{Introduction}

Let $G$ be a discrete group of isometries of the hyperbolic $n$-space $\mathbb{H}^{n}$ with $n \geq 2$. When we take $B^{n}=\left\{x \in \mathbb{R}^{n}:|x|<1\right\}$ as the model space of $\mathbb{H}^{n}$, we can visualize the sphere at infinity of $\mathbb{H}^{n}$ as $S^{n-1}=\left\{x \in \mathbb{R}^{n}:|x|=1\right\}$. And the horospherical limit set $L^{h}(G)$ of $G$ is defined as follows;

$$
\begin{aligned}
L^{h}(G)= & \left\{p \in S^{n-1}: \text { for every horosphere } S \text { in } B^{n}\right. \text { based } \\
& \text { at } p, \text { there is a } g \in G \text { such that } g(0) \in S\},
\end{aligned}
$$

where 0 is the origin of $\mathbb{R}^{n}$. Recently, D. Sullivan obtained several deep results on this horospherical limit set in [5]. In particular, he showed that $L^{h}(G)$ has full measure on $S^{n-1}$ if and only if $G$ is conservative on $S^{n-1}$ ([5, IV. Theorem IV]). If $G$ is ergodic on $S^{n-1}$, then $G$ is conservative, as he noted in [5, IV. Note]. And one may think that ergodicity is equivalent to conservativity (cf. [1, Introduction]). But this is false. In fact, the main purpose of this note is to show the following

THEOREM. For every $n(\geq 2)$, there is a discrete group $G$ of isometries of $\mathbb{H}^{n}$ which is conservative but not ergodic on $S^{n-1}$.

\section{Construction. First we note the following ${ }^{1}$}

LемMA $1([4], \S 4)$. For every $n(\geq 2)$, there is a torsion-free discrete group $G_{0}$ of isometries of $\mathrm{H}^{n}$ such that $M=\mathbb{H}^{n} / G_{0}$ is a compact manifold containing mutually disjoint compact (totally geodesic) submanifolds $N_{1}, N_{2}$ and $N_{3}$ of codimension one such that $M-\left(N_{1} \cup N_{2} \cup N_{3}\right)$ is connected.

Remark. When $n=2$, we can take as $M$ a compact surface of genus 3 , and as $\left\{N_{k}\right\}_{k=1}^{3}$ mutually disjoint loops in a canonical homology base of $M$.

\footnotetext{
'The author wishes to thank Professor S. Kojima (Tokyo Institute of Technology) for teaching him about Millson's work.
} 
Fix such $G_{0}$ as in Lemma 1, and let $\boldsymbol{M}^{\prime}$ be the compact manifold with boundary obtained from $M-\left(N_{1} \cup N_{2} \cup N_{3}\right)$ by attaching six borders $\left\{N_{k}^{+}, N_{k}^{-}\right\}_{k=1}^{3}$ corresponding to $\left\{N_{k}\right\}_{k=1}^{3}$. Next, consider copies $\left\{M^{\prime}(h, i, j)\right\}_{h, i, j \in Z}$ of $M^{\prime}$, and gluing canonically the border $N_{k}^{+}$of $M^{\prime}(h, i, j)$ to that of $N_{k}^{-}$of $M^{\prime}\left(h+\delta_{1 k}, i+\delta_{2 k}, j+\delta_{3 k}\right)$ for every $h, i, j$ and $k$, where $\delta_{m k}$ is Kronecker's delta. Then we have a complete hyperbolic manifold $M_{1}$, which is a torsion-free abelian cover of $M$ of rank 3 . Equivalently, we have a normal subgroup $G_{1}$ of $G_{0}$ such that $M_{1}=\mathbb{H}^{n} / G_{1}$ and $G_{0} / G_{1}$ is a torsion-free abelian group of rank 3 .

Next let $N$ be the submanifold of $M_{1}$ of codimension one corresponding to $N_{1}^{+}$ of $M^{\prime}(0,0,0)$, and fix a positive integer $K$ greater than one. Using $K$ copies of $M_{1}-N$, we can construct in the same way as above, an abelian cover $M_{2}$ of $M_{1}$ of order $K$, i.e. a normal subgroup $G_{2}$ of $G_{1}$ such that $M_{2}=\mathbb{H}^{n} / G_{2}$ and $G_{1} / G_{2}$ is isomorphic to $\mathbb{Z} / K \cdot \mathbb{Z}$, which is a desired group as is shown in the next section.

\section{Proof of theorem}

Let $\pi_{1}$ be the natural projection of $M_{2}$ to $M_{1}$ and $T$ be an isometry of $M_{2}$ of order $K$ such that $\pi_{1} \circ T=\pi_{1}$. Then attaching $K$ points $\left\{e_{k}\right\}_{k=1}^{K}$ to $M_{2}$, we have the Kerékjártó-Stoilow's compactification $\bar{M}_{2}=M_{2} \cup\left(\bigcup_{k=1}^{K}\left\{e_{k}\right\}\right)$ of $M_{2}$ such that $T$ can be extended to an automorphism of $\bar{M}_{2}$ by setting $T\left(e_{k}\right)=e_{k+1}$ for every $k$ (with $\left.e_{K+1}=e_{1}\right)$.

Let $\pi_{2}$ be the natural projection of $\mathbb{H}^{n}$ to $M_{2}=\mathbb{H}^{n} / G_{2}$. Fix $x \in \mathbb{H} \mathbb{H}^{n}$, and set

$$
\begin{aligned}
E_{k}(x)= & \left\{p \in S^{n-1}: \text { letting } L(x, p)\right. \text { be the geodesic ray } \\
& \text { from } x \text { tending to } p, \pi_{2}(L(x, p)) \text { converges } \\
& \text { to } \left.e_{k} \text { in } \bar{M}_{2}\right\}
\end{aligned}
$$

for every $k$. Then we have the following

Lemma 2. $E_{k}(x)$ is a $G_{2}$-invariant measurable set not depending on $x$.

Proof. First we show that $E_{k}(x)$ does not depend on $x$. For any other $x^{\prime} \in$ $H^{n}, \pi_{2}(L(x, p))$ and $\pi_{2}\left(L\left(x^{\prime}, p\right)\right)$ are mutually asymptotic for every $p \in S^{n-1}$. Hence we can see that $p \in E_{k}(x)$ if and only if $p \in E_{k}\left(x^{\prime}\right)$.

In particular, $E_{k}(x)=E_{k}(g(x))$, or equivalently $E_{k}(x)=g^{-1}\left(E_{k}(x)\right)$ for every $g \in G_{2}$; and since measurability of $E_{k}(x)$ is routine, we have the assertion.

In the sequel, we will write simply $E_{k}$ instead of $E_{k}(x)$.

LEMMA 3. It holds that

$$
\begin{gathered}
E_{k} \cap E_{k^{\prime}}=\varnothing \quad \text { if } k \neq k^{\prime}, \\
m\left(E_{k}\right)>0 \text { for every } k \text {, and } \\
m\left(S^{n-1}-\bigcup_{k=1}^{K} E_{k}\right)=0,
\end{gathered}
$$

where $m$ is the canonical measure on $S^{n-1}$.

In particular, $G_{2}$ is not ergodic on $S^{n-1}$. 
Proof. It is clear that $E_{k} \cap E_{k^{\prime}}=\varnothing$ if $k \neq k^{\prime}$. Let $g^{*}$ be an element of $G_{1}$ such that the class of $g^{*}$ in $G_{1} / G_{2}$ corresponds to $T$, then $g^{*}\left(E_{k}\right)=E_{k+1}$ for every $k$, hence $m\left(E_{k}\right)>0$ if and only if it does so for some $k$.

Now for every $p \in S^{n-1}-\bigcup_{k=1}^{K} E_{k}$, we can see from the definition that there is a compact set $A$ in $M_{2}$ and a sequence $\left\{x_{n}\right\}_{n=1}^{\infty}$ of points on $L(x, p)$ tending to $p$ such that $\pi_{2}\left(x_{n}\right) \in A$ for every $n$, which in turn implies that there is a sequence $\left\{g_{m}\right\}_{m=1}^{\infty}$ of mutually distinct elements of $G_{2}$ such that the hyperbolic distances between $g_{m}(0)$ and $L(x, p)$ form a bounded sequence. Hence by definition, $p$ belongs to the conical limit set $L^{c}\left(G_{2}\right)$ of $G_{2}$.

On the other hand, since $M_{1}$ admits Green's function by [3, Theorem 4], hence since $M_{2}$ does so, we can see that $m\left(L^{c}\left(G_{2}\right)\right)=0$ by [5, Corollary III] (cf. [2, VII.7 and VII.8 Theorem 1]). Thus we have

$$
m\left(S^{n-1}-\bigcup_{k=1}^{K} E_{k}\right) \leq m\left(L^{\mathrm{c}}\left(G_{2}\right)\right)=0,
$$

hence $m\left(E_{k}\right)>0$ for every $k$. Since every $E_{k}$ is $G_{2}$-invariant by Lemma 2, $G_{2}$ is not ergodic on $S^{n-1}$.

Now we say that a $G_{2}$-invariant set $E$ on $S^{n-1}$ is non-decomposable if either $m\left(E^{\prime}\right)=0$ or $m\left(E-E^{\prime}\right)=0$ for every $G_{2}$-invariant subset $E^{\prime}$ of $E$. Then we have the following

LEMMA 4. Every $E_{k}$ is non-decomposable.

Proof. Assume that $E_{k}$ is decomposable, and let $E$ be a $G_{2}$-invariant subset of $E_{k}$ such that $m(E)>0$ and $m\left(E_{k}-E\right)>0$. Set $F=\bigcup_{k=1}^{K}\left(g^{*}\right)^{k}(E)$, where $g^{*}$ is as in the proof of Lemma 3, then $F$ is $G_{1}$-invariant, $m(F)>0$ and $m\left(S^{n-1}-F\right)>0$. Then the Poisson's integral

$$
f(t)=\int_{F}\left(\left(1-|t|^{2}\right) /|t-y|^{2}\right)^{n-1} d m(y)
$$

induces a non-constant bounded harmonic function on $M_{1}$ (cf. [2, Theorem V.9]), which contradicts [3, Theorem 1].

LEMMA 5. $m\left(\left(S^{n-1}-L^{h}\left(G_{2}\right)\right) \cap E_{k}\right)=0$ for every $k$. Moreover $G_{2}$ is conservative on $S^{n-1}$.

Proof. Suppose that $m\left(\left(S^{n-1}-L^{h}\left(G_{2}\right)\right) \cap E_{k}\right)>0$ for some $k$. Then by [5, IV. Theorem III], this set is contained in the dissipative part of $G_{2}$, hence is decomposable (which follows at once from the definition of the dissipative part). This contradicts Lemma 4, and we have the first assertion.

Moreover, Lemma 3 and the first assertion imply that $m\left(S^{n-1}-L\left(G_{2}\right)\right)=0$, hence the second assertion follows by [5, IV. Theorem IV].

Remark. We have constructed a group $G_{2}$ such that $S^{n-1}$ is divided into a set of measure zero and $K G_{2}$-invariant sets of positive measure. The author conjectures that a group $G^{*}$ is conservative on $S^{n-1}$ if and only if $S^{n-1}$ is divided into a set of measure zero and (at most countable) non-decomposable $G^{*}$-invariant sets of positive measure. 


\section{REFERENCES}

[1] S. Agard. A geometric proof of Mostew's rigidity theorem for groups of divergence type. Acta Math. 151 (1983), 231-252.

[2] L. Ahlfors. Möbius transformations in several dimensions. Ordway Prof. Lectures in Math. (1981).

[3] T. Lyons \& D. Sullivan. Function theory, random paths and covering spaces, J. Diff. Geom. 19 (1984), 299-323.

[4] J. Millson. On the first Betti number of a constant negatively curved manifold. Ann. of Math. 104 (1976), 235-247.

[5] D. Sullivan. On the ergodic theory at infinity of an arbitrary discrete group of hyperbolic motions, Ann. of Math. Studies 97 (1981), 465-496. 